\title{
Solid pseudopapillary tumour of the pancreas in pregnancy - a case report and literature review
}

\author{
T A Motsepe, ${ }^{1} \mathrm{MB}$ ChB; A Mokhtari, ${ }^{1} \mathrm{MD}$, FRCS, MMed (Chir); N Releni, ${ }^{2} \mathrm{MB}$ ChB; R F Mahabane, ${ }^{3} \mathrm{MB}$ ChB; \\ M C Khaba, ${ }^{2}$ MB ChB, FC Path (SA) Anat, MMed (Anat Path), Dip For Med (SA) Path
}

${ }^{1}$ Department of General Surgery, Dr George Mukhari Academic Hospital, Sefako Makgatho Health Sciences University, Pretoria, South Africa ${ }^{2}$ Department of Anatomical Pathology, National Health Laboratory Services, Sefako Makgatho Health Sciences University, Pretoria, South Africa ${ }^{3}$ Department of Obstetrics and Gynaecology, Dr George Mukhari Academic Hospital, Sefako Makgatho Health Sciences University, Pretoria, South Africa

Corresponding author: M C Khaba (ckhaba@yahoo.co.uk)

\begin{abstract}
Solid pseudopapillary pancreatic tumours (SPTs) are tumours with a low malignant potential though they may metastasise. They are rare in pregnancy and their accelerated growth during pregnancy could be life-threatening. The challenge remains as to how best to treat pregnant patients diagnosed with or suspected of having these tumours. We report a case of a 28 -year-old pregnant female patient at 20 weeks' gestation who presented with mild abdominal pain and an abdominal mass. Physical examination showed an asymmetrical abdomen with associated mild tenderness. Radiological imaging showed a right-sided retroperitoneal tumour. Surgical management was deferred until the pregnancy was at full term. At term, once a live baby had been delivered, excision of the mass was performed. Histopathological assessment showed a solid pseudopapillary tumour of the pancreas. A multidisciplinary approach to a pregnant patient diagnosed with SPT is necessary to ensure the best outcome for both mother and child.
\end{abstract}

S Afr J Obstet Gynaecol 2020;26(1):35-37. https://doi.org/10.7196/SAJOG.2020.v26i1.1562

Solid pseudopapillary pancreatic tumours (SPTs) are relatively uncommon tumours accounting for $1-2 \%$ of all pancreatic tumours ${ }^{[1]}$ and $10-15 \%$ of all pancreatic cystic neoplasms. ${ }^{[1,2]}$ They are considered benign, or tumours with low-malignant potential. ${ }^{[3]}$ Although these tumours potentially have a low malignancy, lymph node metastases are usually found in $2 \%$ of all cases while distant metastases are found in $5-10 \%$ of all cases. ${ }^{[4]}$ SPTs commonly affect women during their second and third decades of life. ${ }^{[2]}$ Most tumours are located in the pancreatic body and tail. The clinical presentation of these tumours is non-specific, and often leads to tumour misdiagnosis. Accurate diagnosis is therefore vital for optimum and effective management. ${ }^{[5]}$ SPT is rare during pregnancy and its accelerated growth can be life-threatening. There are only a few reports of SPTs recorded and resected during pregnancy. Progesterone receptors are regularly identified in SPTs, and the fact that levels of progesterone dramatically increase during pregnancy predisposes them to rupture. ${ }^{[4]}$ The main goal of treatment is to minimise both maternal and fetal risk. Therefore how best to manage a pregnant patient diagnosed with SPT is a specific major challenge. ${ }^{[4]}$

\section{Case}

A 28-year-old female patient presented at 20 weeks' gestation with a large intra-abdominal mass that had been present for 8 years. The patient was initially seen at the local hospital in Limpopo province where magnetic resonance imaging (MRI) showed a right intraperitoneal tumour measuring $16 \times 13 \times 17 \mathrm{~cm}$. She was referred to Dr George Mukhari Academic Hospital and seen by the surgicalgynaecology/obstetrics team. She had presented with mild abdominal discomfort with normal bowel habits and reported good fetal movements. On examination, the abdomen was asymmetrical with prominence over the right hypochondrium. A mass with ill-defined borders was palpated with associated mild tenderness. At this stage, the clinical differential diagnosis included phaeochromocytoma, intra-abdominal sarcoma and complex cysts of the mesentery. Serological investigations were unremarkable. However, carbohydrate antigen 19-9 (CA 19-9) and alpha-fetoprotein (AFP) measurements were slightly raised, which were nonspecific and non-contributory to the diagnosis; values may be altered in pregnancy, making interpretation difficult. HIV serology test was non-reactive. Ultrasound showed a large well-defined heterogeneous mass with multiple areas of necrosis that was located superomedially to the right kidney. MRI (Figs 1A and 1B) demonstrated a well-circumscribed and encapsulated large intraperitoneal mass measuring $16 \times 13 \times 17 \mathrm{~cm}$. There was no loco-regional lymphadenopathy. A gravid uterus was also seen.

At 35 weeks' gestation, she started complaining of a dull pain which progressed steadily in intensity. This case was discussed among the surgical and obstetrician teams and a caesarean section was favoured over normal vaginal delivery. Two doses of betamethasone $12 \mathrm{mg}$ intramuscular injection (IMI) 12 hours apart were given to aid with fetal lung maturation. A caesarean section was done 24 hours later under general anaesthesia. A male infant who weighed $2600 \mathrm{~g}$ was delivered with Apgars of 5/10 and 7/10. The baby was admitted for 24 hours and responded satisfactorily to nalaxone and oxygen therapy.

Once the baby had been delivered, the surgical team commenced with the tumour resection. The tumour was identified and thoroughly examined. It had fibrotic attachments to the duodenum, caudate lobe and segment 5 and 6 of the liver. It was adherent to most of the gallbladder and the porta hepatis of the liver. Careful 


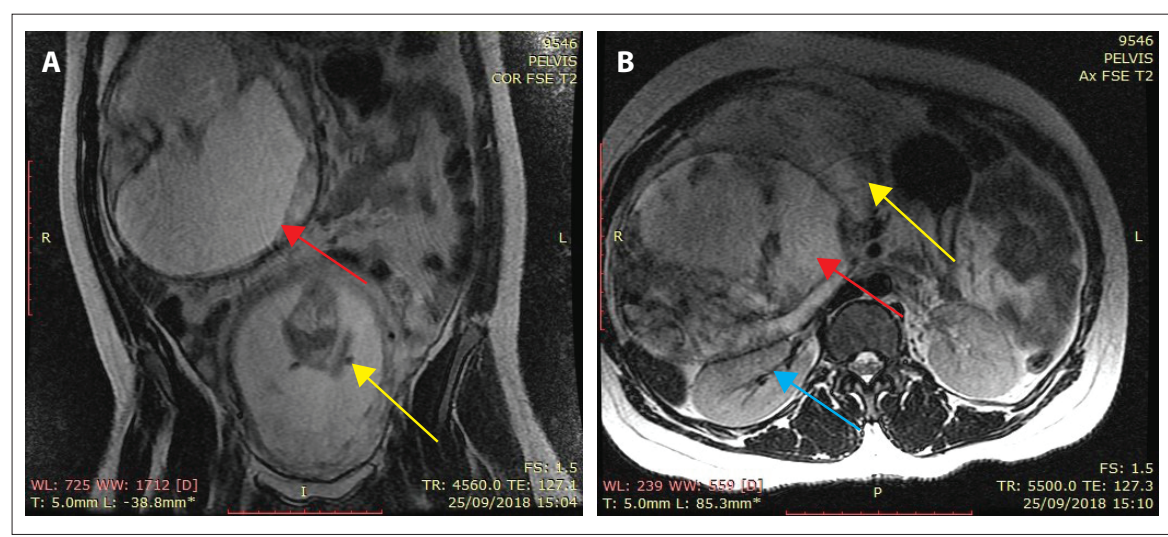

Fig 1. (A) Large encapsulated tumour (red arrow) located above a pregnant uterus (yellow arrow); (B) tumour with solid and cystic areas (red arrow) and the kidney is structurally deformed (blue arrow) as a result of compression.

dissection was done and the tumour removed. The tumour was circumscribed with an intact capsule measuring $16 \times 13 \times$ $17 \mathrm{~cm}$.

On histopathological assessment, the tumour was solid and cystic with haemorrhagic and necrotic areas on cut section. Haematoxylin and eosin (H\&E) sections demonstrated a compressed pancreas with a tumour arranged in nests, solid sheets and pseudopapillae. The tumour cells were round and oval with eosinophilic cytoplasm and eccentrically placed nuclei. The nuclei had fine chromatin with nuclear grooving. Immunohistochemical stains showed positive beta-catenin, CD10, synaptophysin and progesterone receptors on the tumour cells while chromogranin and oestrogen receptors were negative. These features confirmed a solid pseudopapillary tumour of the pancreas. The patient's condition improved gradually until she was discharged from the hospital on day 8 post surgery.

Adjuvant therapy was not offered to the patient as complete resection was achieved.

\section{Discussion}

SPT is a rare condition. It is more common in women than men and may occur in women of childbearing age. Huang et al..$^{[3]}$ found only ten reported SPT cases that had been treated during pregnancy. These tumours generally have a low malignancy potential with a good prognosis. However, local invasion and distant organ and lymph node metastases have been reported. ${ }^{[2,6]}$ SPTs commonly occur in the pancreatic tail, although they have been reported in the head and body. ${ }^{[5]}$ They present with nonspecific features/symptoms which makes diagnosis difficult. ${ }^{[3,6]}$ Some symptoms are as a result of the tumour compressing the adjacent pancreas and include vomiting, nausea, and jaundice. Although the symptoms are nonspecific, abdominal discomfort/pain and a mass are the commonest features. ${ }^{[6]}$ The pain and a mass without other symptoms. The differential diagnosis for these signs and symptoms is vast and not diagnostic. The diagnosis of this tumour relies more on radiolological features as there are no consistent specific tumour markers. ${ }^{[5]}$ AFP and CA 19-9 were unhelpful in the diagnosis of the tumour in this patient, as altered levels during pregnancy may be confusing. However, definite diagnosis is made on histology. It is still not clear whether SPT directly affects pregnancy by compressing the expanding uterus and/or causing fetal compromise. There was no evidence of compromise to pathogenesis includes the mutation of betacatenin which is shown by nuclear staining of the tumour cells which is positive. ${ }^{[2]}$

A raised level of progesterone has been suggested to be associated with tumour growth and/or rupture. ${ }^{[2]}$ Hence, rapid growth may occur in pregnancy. In this patient, the SPT mass was reported to have been present for 8 years, and it would seem that rapid growth occurred during pregnancy. There is still no consensus between different authors regarding the possible role of progesterone. However, it has been suggested that progesterone levels be used to monitor the potential for growth of this tumour in pregnancy, and subsequently as an indicator by the surgeon for excision. ${ }^{[4]}$ The index patient did not have tissue diagnosis until post-surgery. Progesterone was not used for index patient presented with mild abdominal the baby during this pregnancy. Part of SPT monitoring of the SPT mass. Although rare, rupture in the non-pregnant woman may be caused by trauma.

SPTs can be detected radiographically using ultrasound, computed tomography (CT) or MRI. ${ }^{[3]}$ Ultrasound and MRI are preferred as they do not have proven adverse effects on the fetus. ${ }^{[3]}$

Grossly, SPTs may be small or large in size. They are well circumscribed with a pseudocapsule. Post-surgery section demonstrates solid and cystic areas with haemorrhage and necrosis. Microscopically, the tumour cells are arranged in nests, tubules and pseudopapillae. The tumour cells have moderate pale eosinophilic cytoplasm with central/eccentrically placed nuclei with normochromasia. ${ }^{[2,3]}$ Perineurial invasion, lymphovascular invasion, invasion of adjacent structures and distant metastasis are used as criteria for malignancy. ${ }^{[2]}$ Positive immunohistochemical stains include beta-catenin, CD10, synaptophysin and progesterone receptors. ${ }^{[3]}$

The management of SPT in pregnancy can be difficult, partly due to lack of proper guidelines and challenges in trying to establish a balance between maternal and fetal wellbeing, as well as deciding the timing of surgical intervention during pregnancy. Different trimesters are associated with different risks to the fetus. The second or third trimester may be the preferred period for surgical intervention. ${ }^{[3]}$ The American College of Obstetricians and Gynecologists in general recommends operating on patients during the second trimester for non-urgent cases. First trimester surgical treatment may be associated with spontaneous miscarriage; operating during the second and third may carry the risk of premature labour. ${ }^{[3]}$ The growing uterus may obstruct access. Maternal and fetal wellbeing must be assessed to determine the optimal timing of surgery ${ }^{[6]}$ In this case the patient presented in the second trimester. She was deemed fit to continue with the pregnancy until full term (37 weeks) with strict monitoring. The patient was delivered at 35 weeks because of worsening pain.

\section{Conclusion}

SPT is rare in pregnancy with only a few reported cases. There are no proper treatment guidelines. The clinical features of SPT are nonspecific and the differential diagnosis should be considered, but 
knowledge of SPT is necessary to inform this differential. The collaboration of different specialities is essential in management. The wellbeing of the mother and baby should always be assessed to avoid morbidity and mortality.

\section{Declaration. None.}

Acknowledgements. The authors thank Dr M E Seleka (Head of Department of Language Proficiency/English at Sefako Makgatho Health Sciences University) for assistance with language editing.

Author contributions. MCK and TAM conceptualised the case report. TAM, $\mathrm{AM}, \mathrm{NR}$ and RFM made equal contributions to writing and revising the manuscript.

Funding. None.
Conflicts of interest. None.

1. Sharma PK, Mehrotra S, Gleisner AL, Schulick RD, McCarter MD. Recurrent solid pseudopapillary neoplasm of pancreas: Case report and review of literature. J Pancreat Cancer 2018;4(1):25-29. https://doi.org/10.1089/pancan.2018.0006

2. MacDonald F, Keough V, Huang WY, Molinari M. Surgical therapy of a large pancreatic solid-pseudopapillary neoplasm during pregnancy. BMJ Case Rep 2014;2014:2-6. https://doi. org/10.1136/bcr-2013-202259

3. Huang T, Zhu J, Zhou H, Zhao A. Solid pseudopapillary neoplasm of pancreas in pregnancy treated with tumor enucleation: Case report and review of the literature. Niger J Clin Pract 2018;21(9):1234-1237. https://doi.org/10.4103/njcp.njcp_39_18

4. Yee AM, Kelly BG, Gonzalez-Velez JM, Nakakura EK. Solid pseudopapillary neoplasm of the pancreas head in a pregnant woman: Safe pancreaticoduodenectomy postpartum. J Surg Case Rep 2015;2015(8):rjv108. https://doi.org/10.1093/jscr/rjv108

5. Lanke G, Ali FS, Lee JH. Clinical update on the management of pseudopapillary tumor of pancreas. World J Gastrointest Endosc 2018;10(9):145-155. https://doi.org/0.4253/wjge.v10.i9.145

6. Tanacan A, Orgul G, Dogrul AB, Aktoz F, Abbasoglu O, Beksac MS. Management of a pregnancy with a solid pseudopapillary neoplasm of the pancreas. Case Rep Obstet Gynecol 2018;2018:1-3. https://doi.org/10.1155/2018/5832341 